\title{
Some Reflections on Rangeland Pasture Trials
}

\author{
David Scott and L. Alex Maunsell
}

$\mathrm{F}$ ield trials are the link between science and practical rangeland management. As one progresses in age from seniority to senility there is a tendency to pontificate on one's experiences in the hope that the next generation may listen. The following is a light-hearted but serious look at the conception, setting down, implementation and analysis of field trials. Beneath each are lessons that have been learned from the hard experience of running our own trials and from observing others running theirs.

\section{'Is it a problem'}

In conception, rangeland problems go through periods of interest, fad, or concern like any other human activity. Our experience is that it is often better to let things 'simmer on the back-burner' for a couple of years before setting-up what may be a long-term trial. That allows time to see whether it really is a problem and to determine the more exact nature of the problem. It also allows time for the researcher to think about how it could be tackled by a field trial, and the possibilities of where, who pays, and who is actually going to do the work.

\section{'What is the question?'}

There will be many possible explanations or permutations of what are the likely basis of a perceived problem, the techniques that are available, and the resources of time and materials that can be allocated. One has to make a pragmatic decision on which combination to run with, and then stick with it. You won't solve the world's problem with one trial. It helps if the question is formally stated and becomes the guideline objective for the particular trial, even if it is conceded that it is only one aspect of a more general question.

\section{'Science or demonstration trial?'}

In the present era of 'outside funding,' technology transfer' and 'on-ranch trials' care is needed in defining what is the priority function of a particular investigation, and what are the different constraints that those may place on its design. Demonstration trials should be largely a scaling-up of an option established by earlier work, in comparison with some previous options that it may replace. In this there is an advantage of prominence, under 'practical' management, and contrasts in close proximity to each other. Science trials should be a jump into the unknown, requiring tighter control of management and treatment factors, requiring at least some treatment combinations outside what may be considered 'practical', randomization, and occa- sionally better done out of the public gaze. We are not convinced that the different requirements of the two types of trials can always be efficiently incorporated within the same trial design, without compromising one or both requirements.

'If you had enough information to design the perfect trial you probably wouldn't need to do it'

A good deal of pragmatism is needed in trial design. Yes, take account of all previous experience, regard for statistical design, and all the things that may go wrong, but take the plunge. An old adage says 'if all else fails do something'. A reasonable trial on the ground is probably more useful than a perfect trial on the drawing board.

\section{'Trials must have a stock-gate'}

If trials are to be relevant to rangeland agriculture, then grazing must be one of the treatments. Yes, there can be exclosure fences for trials to establish pastures, or for ground revegetation. But if the results are to be relevant to general rangeland agriculture, then there has to be a gate in the fence, which is used from time to time, and you have a record of what goes through it.

\section{'Contrasts give perspective'}

Don't be frightened by the number of treatments. Many contrasting treatments allow one to obtain a better perspective on particular results. Compare this with some trials, particularly by commercial companies, that only feature their one new product. One can never be sure whether the response is environment, site or product.

'Large enough to be relevant-small enough to see com parisons'

A rancher will probably never get excited about a square meter plot, irrespective of how spectacular are the resulting numbers. Conversely, research stations and other groups develop large areas, and while ranchers are impressed on a visit, tend to dismiss them when they go home- 'they have just got a good bit of dirt'. The need is to have plots or trials large enough so that they are seen as potentially applicable to paddocks or ranches, yet small enough so that comparisons can still be seen in conjunction with previous or other alternatives within the same context, or from the same viewing point. Our experience in the New Zealand high-country pasture trials, was that a working compromise between the two demands were plot sizes in the range of 
0.1-0.2 ha each, and with a proportion of these left in their unimproved state.

\section{'Go in for the long haul'}

If trials are to be relevant to rangeland farmers then they should be of a similar time scale as farming. Many trials start with 'a hiss and a roar' in the first flush of enthusiasm, and then peter out. Rangeland trial literature is over-burdened with germination and establishment studies, but very sparse for trials that have run for more than 3-4 years- the time scale that ranchers would really like to know what the differences are. There is also enough experience about to indicate that the early results may not be a very good predictor of long term effects, and that many effects take time to develop.

\section{'Use time-don't fight it'}

This is the same sentiment expressed in a slightly different manner, particularly as it relates to measurements. Our experience is that trends which may require very intensive sampling to be detected within short time frames, become relatively easy if one is patient and waits for a longer time frame. For example the need to detect a $1 \%$ difference in a monthly time frame, becomes a need to only detect a $10 \%$ difference on a yearly time frame. Longer time frames also more directly answer the questions about sustainability.

\section{'What sort of answer do you want'}

Before one decides on what measurements to make one needs to decide on what sort of answers will satisfy the question asked. A practical suggestion is, even at the trial design stage, to make a mock-up of possible tables and figures that might appear in the final report or paper, and how, given different scenarios, they would be used. These help focus on what measurements are necessary.

\section{'Sampling efficiency'}

If one took a dispassionate view on how science and trial results were finally used in practice, then predominately it is only as a qualitative or value judgement. For example 'that Kara wheat-grass is better than Currie wheat-grass' in such-and-such a situation. It is almost never asked or used 'how much better?'-although that is what is initially reported. As many results are only ultimately used in only a qualitative or value judgement sense, we suspect that there could be great gains in the efficiency in sampling of trials. This is by more frequent use of non-parametric and ranking techniques, even though these are commonly regarded as being 'imprecise' and 'not scientific'. The gains in efficiencies at that stage free resources for other monitoring.

Sampling efficiency is about accuracy obtained for time spent. A reduction in time needed to get one observation means that more observations can be made and more land types sampled. This is particularly relevant to rangeland, which almost by definition, is variable.
'Establish a minimum, not maximum, set of measure ments for each trial'

This is the same sentiment expressed in a slightly different manner, but focuses on what is the absolute minimum measurement that would be needed to answer the question. This should be seen as a means of determining priorities rather than skimping on work. Also, as the next section will indicate, it allows time to see whether a particular trial 'works'. Too many trials start with grandiose proposals to measure everything under the sun, and then become locked into commitment of time and resources, irrespective of how the trial develops. Our experience is that it is generally better to start modestly, and go big if the early results are favorable, rather than start big and crash.

For a series of high-country pasture trials we ran over 20-30 years, each of 3-18 years duration, the minimum set of measurements in many were: the species pasture composition of each treatment plot in late spring, after a common regrowth period from mid-winter; and the annual animalgrazing-days achieved on each plot.

\section{'Multiple trials and hedge your bets'}

Field trials will often be, or should be, a step into the unknown. If you are not trying to push the boundaries then it is a demonstration or technology trial rather than a science trial. It must be expected that trials will not behave as planned, and this must be allowed for in their design. Our experience is that this can be accommodated in two ways.

The first is by a multiple variable approach, of alternative forms or approaches to the main factors, either as several small trials of particular treatment combinations, or as large initial multiple treatment trials that may be separated into sections as the trial progresses.

The second is by starting with only a minimum set of required measurements so as not to initially over-commit resources, and to wait ('use time-don't fight it') to see how each component develops, before deciding where to invest further intensive measurement.

\section{'The last person you should consult is a statistician'}

This is intended as a reminder to the proposer, rather than a criticism of statisticians. The proposer of trials should already have sorted out where are the likely sources of variation within a site, what are the appropriate plot sizes and edge effects, what treatments have to be blocked because of machinery or grazing an-

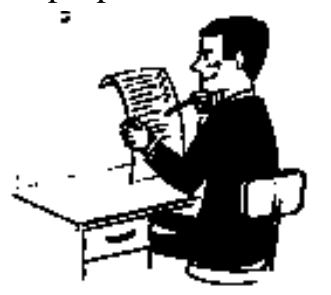

imal constraints, etc., etc. These are all topics that the proposer should have considered. It is only when those topics are in hand that a statistician can help in design of the most efficient layout to answer the question proposed.

There has been a touching belief in the past that statisticians were the high priests of trial design, and could produce results out of any numbers you dropped on their desk. In this day and age, that mantle of invincibility seems to be 
passing from the statisticians to the modelers, so again they may need to be consulted, but only after you have thought through the implication in both a science and practical management sense of alternatives that they may suggest.

\section{'Replicate treatments not space'}

Replication is the corner stone of experimental design for statistical analysis, as a means of identifying known sources of assumed random variation. Our view is that a vast amount of the rangeland and agricultural literature has given undue emphasis to the question of spatial replication to overcome concerns about environmental variability, and under-emphasis on the true need to replicate treatment effects. The source of such variation may be very low in the weighing out of fertiliser using analytical reagents and precision balances, but the field application of the same may well have a spatial variability of $5-20 \%$. One of the disconcerting experiences in field trials is when you have a particular cultivar, using seed from different sources, and find differences that in other contexts are ascribed to differences between cultivars or species. Would only one source of 'farm yard manure' be acceptable in a trial comparing its efficacy relative to artificial nitrogen fertiliser?

In practice, replication is no different from other treatment variables. A statistician, given data with treatments labeled only A, B, C etc. has no way of determining which were the replicates. The form of the statistical analysis will be almost wholly dependent on what we assume to be the type of variation in each of the treatments (i.e. random or fixed), and with those assumptions seldom verifiable.

In practice there is much more need to have replication of treatments as well as spatial replication, and clearer statement of our assumptions about the assumed variability in each treatment. For example whether a cultivar is a random sample from an infinite population of species, a random sample from the limited population of named cultivars, or only one of a few cultivars in the particular trial?

\section{'How many reps are enough'}

The short answer is-always more than practical. Statistical theory is about the behavior of a large number of numbers. Even small sample theory is thinking of $10-30$. Precedent from the statisticians of the back-of-thematchbox and adding machine era, have let us get away with murder with only 2-4 replications. While these

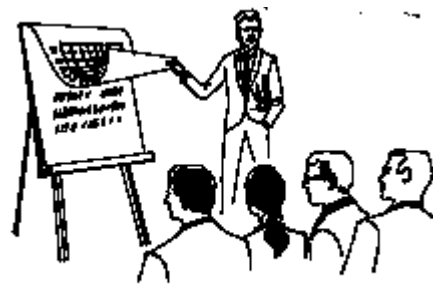
may be within the letter of statistical theory, they are certainly well below the spirit of the theory.

\section{'A room with a view'}

Besides the science requirements of uniformity (or known variability) of site and plot layout, there is great ad- vantage for public relation purposes, or explaining trials to groups of visitors, that there are one or a few vantage points from which the whole trial can be viewed.

\section{'Experimental layout is not about geography'}

As indicated above, we are concerned that replication is taken to mean spatial replication rather than treatment replication, and the extent to which trials are judged from the dispersion of treatments within a plot plan without reference to the actual land. Few textbooks on experimental design refer to a necessary earlier stage, before plot allocation, of assessing the various covariates or attribute of each potential plot, and assigning them into like groups. It is the random allocation of treatments within such like groups that is the important decision, not their actual location. Our experience is that this initial phase of assessment of attributes of each potential plot, however crude, leads to better plot layouts, and can be among the main explanatory variables at the analysis stage.

\section{'Remember the past'}

Every site has its own unique past. Many field trials, particularly on research stations, are superimposed on areas used for previous trials. Never under-estimate the effect of this past on present trials. In some instances this past can be taken as the base from which new increases are being attempted. However in other cases, past effects may negate the context of a present trial e.g. any past fertiliser can negate a 'zero fertiliser' treatment. In practice more use can be made of past trial effects by inclusion as plot covariates to be adjusted for in interpreting a current trial.

'You will probably be judged for your management skills before your science break-through'

Visitors to a trial, particularly ranchers, will be making unconscious assessments even before you open your mouth e.g. rickety fences, scouring cattle etc. The effort spent on such routine considerations, means that you at least get listened to.

\section{'Maintain the treatments'}

In the management of trials, the first priority on all occasion should be to maintain the treatments-if that means going out to shift stock in a snow storm so-be-it. Insistence on this requirement give trials continuity, and with continuity, confidence. Also, the insistence that this is the priority makes day-to-day work decisions easier.

\section{'Expect the unexpected'}

Good science trials should be largely an exploration into the unknown. As said earlier if we knew enough to design a perfect trial, we probably would not need to do it. The implication of this is that very few trials will run as predicted. A few may be spectacularly successful, many will not achieve what was hoped for, and yet others will give completely unexpected results. Probably only a quarter of field 
trials produce really useful results. Resources in time and materials must allow for this uncertainty.

One of the reasons for establishing only a minimum set of measurements at the inception of a range of trials is that it builds in a reserve of resources of time and materials. These then can be concentrated on measuring useful parameters of trials that are 'working'-either as expected, or when they give unexpected new responses.

In trial supervision there should be the combination of: firstly insistence on the priority of maintaining the treatments, secondarily on recording the minimum measurement set, and thirdly the readiness to pour resources into trials that are working. This allows multiple trials to be run simultaneously, with the added perspective that gives.

\section{'Be a record fanatic'}

Have plots marked, sample bags double labeled, make copious notes, date them, and file them. The human brain does not keep very accurate records. While it is obvious this evening that you have just spent the day taking 8 soil cores to $8 \mathrm{~cm}$ depth per plot, will you be so sure next week, and will you remember these details in 10 years time when the results need to be worked up? Was it 'sulphur super extra' superphosphate or 'maxi' superphosphate that was put on in 1985, and what was their exact composition in that year?

While much of the information is never used, it is continually surprising what obscure little fact becomes critical in understanding the final outcome. Think now of all the grass trials over the century that have reduced values because there are no voucher specimens to accommodate changes in understanding of taxonomy or fungal endophyte level.

'Qualitative treatment variables at the design stage should have been replaced by measured quantitative variables by the analysis stage,

Many field trials make poor use at the analysis stage of the data on which a lot of time and effort has been spent in getting. The analysis of field trials is dominated by the analysis of variance approach where the treatments and their levels are treated as only qualitative mutually exclusive classes. In practice many of them are quantitative (e.g. fertiliser levels), or can be made quantitative or ordinal (e.g. 'low' and 'high' stocking rates to actual stocking rate), thereby allowing more powerful regression-like analysis methods to be applied to the results.

\section{'Your mother does not work here'}

As a post-script, clean up afterwards. The countryside is littered with derelict trials of rank growth, broken down fences, and rotting pegs. They do nothing for the image of those that put them in, or for the following generation of researchers.

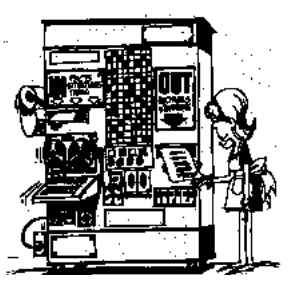

'If you don't finish and report, you may as well not have started'

If field trials are to add to rangeland knowledge, then there must be some way for people to know they existed and at least what were the main results. If they do not reach at least that stage, then they may as well have never been started, and may be condemning some one to repeat the same mistakes, and go up the same blind alley.

Papers, as in this journal, or the Journal of Range Management, are the common method of recording such trials. But these are biased towards successful trials, both in terms of completion and magnitude of effects. For rangelands we have not really developed a good method for archiving records of all trials, particularly those that were ambivalent or negative in their results. Think of all the data that has disappeared in recent amalgamations and restructuring of rangeland science in different countries.

There probably can be no general conclusion. This paper has given two persons' experiences and views on how to conduct rangeland field trials. Hopefully many of the points are in accordance with the experience of others, and may provide some guidance for other researchers.

Authors were scientists with AgResearch, Lincoln, New Zealand. 